Correspondence

Sanjeev K. Sahni

Sanjeev_Sahni@

urmc.rochester.edu

Received 3 November 2006

Accepted 15 March 2007

\section{Comparative analysis of host-cell signalling mechanisms activated in response to infection with Rickettsia conorii and Rickettsia typhi}

\author{
Elena Rydkina, ${ }^{1}$ Abha Sahni, ${ }^{1}$ David J. Silverman ${ }^{2}$ and Sanjeev K. Sahni ${ }^{1}$ \\ ${ }^{1}$ Hematology-Oncology Unit, Department of Medicine, University of Rochester School of Medicine \\ and Dentistry, Rochester, NY 14642, USA \\ ${ }^{2}$ Department of Microbiology and Immunology, University of Maryland School of Medicine, \\ Baltimore, MD 21201, USA
}

\begin{abstract}
The Gram-negative intracellular bacteria Rickettsia conorii and Rickettsia typhi are the aetiological agents of Mediterranean spotted fever and endemic typhus, respectively, in humans. Infection of endothelial cells (ECs) lining vessel walls, and the resultant vascular inflammation and haemostatic alterations are salient pathogenetic features of both of these rickettsial diseases. An important consideration, however, is that dramatic differences in the intracellular motility and accumulation patterns for spotted fever versus typhus group rickettsiae have been documented, suggesting the possibility of unique and potentially different interactions with host cells. This study characterized and compared $R$. conorii- and $R$. typhi-mediated effects on cultured human ECs. The DNA-binding activity of nuclear transcription factor $-\kappa \mathrm{B}(\mathrm{NF}-\kappa \mathrm{B})$ and the phosphorylation status of stress-activated p38 kinase were determined as indicators of NF- $\kappa$ B and p38 activation. $R$. conorii infection resulted in a biphasic activation of NF- $\kappa \mathrm{B}$, with an early increase in DNA-binding activity at $3 \mathrm{~h}$, followed by a later peak at $24 \mathrm{~h}$. The activated NF- $\kappa \mathrm{B}$ species were composed mainly of RelA p65-p50 heterodimers and p50 homodimers. R. typhi infection of ECs resulted in only early activation of NF- $\kappa \mathrm{B}$ at $3 \mathrm{~h}$, composed primarily of p65-p50 heterodimers. Whilst $R$. conorii infection induced increased phosphorylation of p38 kinase (threefold mean induction) with the maximal response at $3 \mathrm{~h}$, a considerably less-intense response peaking at about $6 \mathrm{~h}$ post-infection was found with $R$. typhi. Furthermore, mRNA expression of the chemokines interleukin (IL)-8 and monocyte chemoattractant protein-1 in ECs infected with either Rickettsia species was higher than the corresponding controls, but there were distinct differences in the secretion patterns for IL-8, suggesting the possibility of involvement of post-transcriptional control mechanisms or differences in the release from intracellular storage sites. Thus, the intensity and kinetics of host-cell responses triggered by spotted fever and typhus species exhibit distinct variations that could subsequently lead to differences in the extent of endothelial activation and inflammation and serve as important determinants of pathogenesis.
\end{abstract}

\section{INTRODUCTION}

Transmitted by various arthropod vectors and responsible for the spotted fever group (SFG) and typhus group (TG) of diseases, rickettsiae are Gram-negative bacteria that display an obligatory intracellular lifestyle and an affinity for infecting vascular endothelium of their human hosts (Valbuena \& Walker, 2006). After internalization,

Abbreviations: EC, endothelial cell; EMSA, electrophoretic mobility shift assay; GAPDH, glyceraldehyde 3-phosphate dehydrogenase; $1 \kappa \mathrm{B}$, inhibitor of $\kappa \mathrm{B}$ protein; IL, interleukin; MAPK, mitogen-activated protein kinase; $\mathrm{MCP}$, monocyte chemoattractant protein; NF- $\kappa \mathrm{B}$, nuclear transcription factor- $\kappa \mathrm{B}$; p.i., post-infection; SFG, spotted fever group; $T G$, typhus group; TNF, tumour necrosis factor. rickettsiae quickly escape from the phagosome into the nutrient-rich cytoplasm, where they either synthesize ATP via the tricarboxylic acid cycle or derive energy from the host cell through an ATP/ADP translocase system (Audia \& Winkler, 2006) and multiply by simple binary fission (Hackstadt, 1996). There also exist, however, distinct differences in the intracytoplasmic behaviour of SFG and TG organisms. For example, Rickettsia rickettsii and Rickettsia conorii, which belong to the SFG, form a polar actin tail by subverting cellular actin dynamics, and use it for movement within the host cytosol and occasionally to traverse through the nucleus (Heinzen, 2003). This actinbased motility mechanism also facilitates their spread from one cell to another and is dependent on the activity of 
RickA, a protein capable of activating the Arp2/3 complex and found to be present in SFG Rickettsia species (Gouin et al., 2004; Jeng et al., 2004). TG rickettsiae, on the other hand, display either short and 'hooked' (Rickettsia typhi) or no (Rickettsia prowazekii) actin tails, probably due to the absence of a functional RickA homologue in their genomes (McLeod et al., 2004). Accordingly, R. typhi exhibits blunted circular motion compared with the straight line path with constant momentum displayed by $R$. rickettsii and $R$. conorii (Gouin et al., 1999; Heinzen et al., 1999; Heinzen, 2003) and, in general, TG rickettsiae tend to accumulate in their host cells to significantly higher numbers than SFG rickettsiae (Hackstadt, 1996, 1998).

Thorough analysis of tissue specimens from human cases of disease and animal models of infection, using $\mathrm{C} 3 \mathrm{H} / \mathrm{HeN}$ mice with $R$. conorii and $R$. typhi, it has been established that vascular endothelial cells (ECs) are the major preferred targets of infection (Olano, 2005; Sahni, 2007; Valbuena \& Walker, 2006). Available experimental evidence further suggests increased expression of surface adhesion molecules (intercellular adhesion molecule-1 and E-selectin), cytokines [interleukin (IL)-1 $\alpha$ ] and chemokines [IL-8 and monocyte chemoattractant protein (MCP)-1] by cultured human ECs infected in vitro with $R$. rickettsii or $R$. conorii (Sporn et al., 1993; Kaplanski et al., 1995; Sporn \& Marder, 1996; Dignat-George et al., 1997; Valbuena et al., 2003; Clifton et al., 2005). Published studies from our laboratory have further identified activation of nuclear transcription factor $-\kappa \mathrm{B}(\mathrm{NF}-\kappa \mathrm{B})$ and a specific mitogen-activated protein kinase (MAPK) module, namely p38 MAPK, as important components of upstream intracellular signalling events responsible for host-cell transcriptional activation in response to $R$. rickettsii infection (Sporn et al., 1997; Shi et al., 1998; Rydkina et al., 2005). A comparative study of interactions of Rickettsia akari (a SFG species that primarily infects perivascular macrophages and causes benign biphasic disease termed rickettsialpox; Boyd, 1997) and $R$. typhi with mouse macrophages revealed noticeable differences in the secretion pattern of tumour necrosis factor (TNF) $-\alpha$, IL- $1 \beta$ and IL- 6 , and mRNA expression of the inhibitor of $\kappa \mathrm{B}$ protein, $\mathrm{I} \kappa \mathrm{B} \alpha$, as an indirect measure of NF- $\kappa \mathrm{B}$ activation (Radulovic et al., 2002). An important aspect of interactions between pathogenic Rickettsia species and host ECs that remains to be addressed in detail is whether different signalling mechanisms contribute to host-cell responses or whether similar upstream events may be activated with either different kinetics patterns or to apparently different degrees after infection with SFG and TG rickettsiae. Thus, taking into consideration the fact that divergent cytopathologies probably result in potentially different interactions with host cells, the present study was designed to define the similarities as well as the differences in endothelial responses to infection with $R$. conorii, the causative agent of Mediterranean spotted fever, and the TG species $R$. typhi, responsible for murine typhus or endemic typhus in humans.

\section{METHODS}

Cell culture. Primary cultures of human umbilical vein ECs from umbilical cords collected within $48 \mathrm{~h}$ of delivery were established as described by Sporn et al. (1991). Cells were grown in McCoy's 5A medium (Flow Laboratories) containing fetal bovine serum (20\%, $\mathrm{v} / \mathrm{v})$, EC growth supplement $\left(50 \mu \mathrm{g} \mathrm{ml}^{-1}\right.$; Collaborative Research), Lglutamine (300 $\mathrm{g} \mathrm{ml}^{-1}$; Gibco) and heparin (100 $\mathrm{g} \mathrm{ml}^{-1}$; Sigma). After a second passage, cells were seeded to achieve about $80-90 \%$ confluence after 5-7 days in culture, at which point they were used for experiments.

\section{Determination of rickettsial viability and in vitro infection of} ECs. The Sheila Smith strain of $R$. rickettsii, a human isolate of unknown passage history, was obtained as a plaque-purified seed stock prepared in Vero C1008 (African green monkey epithelial) cells (ATCC). R. conorii (Malish 7) and R. typhi (Wilmington strain) were obtained from Dr G. Dasch (Centers for Disease Control and Prevention, Atlanta, GA, USA). Infectious stocks for both species of rickettsiae used in our study were obtained from heavily infected Vero cell monolayers by a combination of differential and density-gradient centrifugation procedures described previously (Sahni et al., 1998). Aliquots of rickettsiae to be used for infection of ECs were processed by plaque assay to determine the titre of viable organisms (p.f.u. $\mathrm{ml}^{-1}$ ) (Rydkina et al., 2004). For R. typhi, the plaque assay procedure was modified to enhance the formation of resultant plaques by inclusion of dextran sulphate (50 $\mathrm{ng} \mathrm{ml}^{-1}$; Sigma) in the agarosecontaining overlay medium and by the performance of all incubations at $35{ }^{\circ} \mathrm{C}$ (Policastro et al., 1996). The determination of extent of infection for different Rickettsia species was performed by real-time PCR using primer pair Rp877p and Rp1258n for the rickettsial citrate synthase gene (Regnery et al., 1991) and a TaqMan-based internal probe with $100 \%$ homology to the target gene. ECs were infected with viable Rickettsia organisms at an m.o.i. of 6. In experiments where infection was performed for durations of more than $3 \mathrm{~h}$, extracellular rickettsiae were removed after $3 \mathrm{~h}$ by aspiration of the initial inoculum; cell monolayers were washed quickly with fresh medium at $37{ }^{\circ} \mathrm{C}$ and further incubation was carried out in fresh culture medium.

Analysis of NF- $\kappa$ B activation by electrophoretic mobility shift assay (EMSA). The DNA-binding activity of NF- $\kappa \mathrm{B}$ was monitored by EMSA. Nuclear protein extracts and double-stranded consensus NF- $\kappa$ B sequence end-labelled with $\left[\gamma^{32} \mathrm{P}\right] \mathrm{ATP}$ via T4 polynucleotide kinase were prepared by following previously published procedures (Sahni et al., 1998, 2003). EMSA was routinely performed with a gelshift assay system (Promega) following the manufacturer's instructions and using $2 \mu \mathrm{g}$ nuclear protein for each experimental sample. After binding reactions, DNA-protein complexes were separated from the free probe by electrophoresis at $100 \mathrm{~V}$ for $2 \mathrm{~h}$ on $4 \%$ nondenaturing, low-ionic-strength polyacrylamide gels prepared in $0.5 \times$ Tris/borate/EDTA buffer. Nuclear extracts from HeLa cells (Promega) were included in each independent assay as a positive control. The gels were vacuum dried at $80^{\circ} \mathrm{C}$ for $1 \mathrm{~h}$ and subjected to radiographic exposure using Kodak Biomax film.

Supershift analysis of activated NF- $\kappa$ B species. Antibodies targeted against p65, p50 and the c-Rel subunits of NF- $\kappa \mathrm{B}$ were purchased from Santa Cruz Biotechnology and used according to our previously described protocols (Sahni et al., 1998, 2003). Briefly, $1 \mu \mathrm{g}$ each specific antibody was mixed into the gel-shift reactions prior to addition of radiolabelled oligonucleotide probe and incubated at $37^{\circ} \mathrm{C}$ for $20 \mathrm{~min}$. All reaction mixtures were then further incubated with labelled probe and analysed by EMSA as described above, with the exception that electrophoresis to resolve gel-shifted and supershifted complexes was carried out at $100 \mathrm{~V}$ for $3 \mathrm{~h}$ or longer. 
Analysis of $1 \kappa \mathrm{B} \alpha$ phosphorylation and p38 MAPK activation by Western blotting. Uninfected (control) and Rickettsia-infected ECs were washed three times with cold PBS and subjected to lysis in an SDS- and DTT-containing Tris buffer supplemented with a broad spectrum of protease and phosphatase inhibitors (Cell Signalling Technology). Total protein lysates thus obtained were kept frozen at $-20{ }^{\circ} \mathrm{C}$ until further analysis. Equal volumes of cell lysates were boiled for $10 \mathrm{~min}$, subjected to SDS-PAGE on $12.5 \%$ denaturing gels, transferred to nitrocellulose membrane and probed with antibodies designed for specific detection of either phospho- $\mathrm{I} \kappa \mathrm{B} \alpha$ (Ser32) or total $\mathrm{I} \kappa \mathrm{B} \alpha$ provided in the PhosphoPlus- $\mathrm{I} \kappa \mathrm{B} \alpha$ (Ser32) antibody kit (Cell Signalling). Positive controls for phospho- and total- $\mathrm{I} \kappa \mathrm{B} \alpha$ provided by the manufacturer were included in each blot. Antibodies for phospho- and total $\mathrm{I} \kappa \mathrm{B} \alpha$ were used at respective dilutions of $1: 2000$ and $1: 500$ in $5 \%$ BSA (w/v). Antibody for phospho-p38 MAPK (Cell Signalling) was used at a dilution of $1: 2000$. In some experiments, antibody for total p38 MAPK (Cell Signalling) was used. Protein lysates of anisomycin-stimulated ECs were used as positive controls for specific detection of p38 MAPK activation. After development with horseradish peroxidase-conjugated anti-rabbit IgG secondary antibodies and a Western Lighting chemiluminescence developing system (Perkin Elmer Life Sciences), blots were exposed to Hyperfilm ECL film (Amersham Biosciences). The relative positions of biotinylated molecular mass markers on each gel were also detected using anti-biotin horseradish peroxidase-conjugated antibody (Cell Signalling).

Analysis of mRNA levels of chemokine genes. RNA isolated from infected and uninfected ECs using TRI Reagent (Molecular Research Center) was subjected to analysis by multiplex RT-PCR using a kit for detection of human chemokine genes (Maxim Biotech). All reactions were performed in the linear range for each transcript [in comparison with a glyceraldehyde 3-phosphate dehydrogenase (GAPDH) reference control] using $4 \mu \mathrm{g}$ RNA. The PCR products obtained from experimental samples and a positive control were resolved simultaneously on a $2 \%$ agarose gel and visualized by ethidium bromide staining. The sizes for GAPDH, MCP-2, MCP-1, Rantes, IP-10 and IL-8 gene products were 500, 330, 277, 233, 199 and 173 bp, respectively. RT-PCR experiments were performed with at least two independent sets of samples with similar results.

Measurement of IL-8 and MCP-1 secretion. Culture supernatants from Rickettsia-infected ECs and simultaneous uninfected control samples were cleared by centrifugation at $10000 \mathrm{~g}$ for $5 \mathrm{~min}$. and stored as $500 \mu \mathrm{l}$ aliquots at $-80{ }^{\circ} \mathrm{C}$. The amount of IL- 8 and MCP-1 protein in the supernatants was measured by ELISA using a Quantikine kit (R\&D Systems) following the manufacturer's instructions. The minimal detectable ranges of IL- 8 and MCP-1 were 1.5$7.5 \mathrm{pg} \mathrm{ml}^{-1}$ and $<5.0 \mathrm{pg} \mathrm{ml}^{-1}$, respectively. For each sample, at least two dilutions were assayed in duplicate and the absorbance values corresponding to the linear range of a standard curve were used for the determination of chemokine concentration.

Scanning and densitometry. Autoradiographs representing optimum exposures of the gels were scanned in greyscale mode using a Hewlett Packard 6300C Scanjet system and the intensities of bands were quantified using IMAGEQUANT software, version 3.3 (Molecular Dynamics). The densities of the tubulin and GAPDH bands were used to correct for variations in loading and to calculate normalized values for the levels of phospho- and total proteins. For comparison, the normalized value of uninfected controls was either assigned an arbitrary value of 1 or was considered to be $100 \%$, and the effect of infection was determined as a function of this baseline level.

Statistical analysis. The values are presented as mean \pm SEM. A pooled two-tailed Student's $t$-test analysis was used to compare the two groups of data and the results were considered to be statistically significant at $P$ values of $\leqslant 0.05$.

\section{RESULTS AND DISCUSSION}

\section{Infection of cultured ECs with $R$. conorii and R. typhi}

Earlier studies from our laboratory demonstrated that cultured ECs are efficiently infected by $R$. rickettsii in vitro and that a host cell : viable rickettsiae ratio of $1: 6$ yields infection of more than $80 \%$ of cells with three or four organisms at $6-8 \mathrm{~h}$. Bearing in mind the requirement to achieve a similar level of intracellular infection with different species, we incubated ECs with $R$. conorii or $R$. typhi at an m.o.i. of 6 for $3 \mathrm{~h}$ (sufficient time considering that in vitro adhesion and invasion is almost instantaneous; Teysseire et al., 1995) and determined the number of intracellular organisms by quantitative PCR using rickettsial citrate synthase gene $(g l t A)$-specific primers and a TaqMan probe to determine the mean copy number of gltA in relation to that of GAPDH. This resulted in host-cell infection with a mean of $2.57 \pm 0.18$ organisms $(n=3)$ for $R$. conorii and $3.22 \pm 0.12$ organisms $(n=3)$ for $R$. typhi. The slightly higher rate of infection with $R$. typhi was probably due to underestimation of the $R$. typhi titre in stock preparations, as plaques generated by TG Rickettsia species tend to be smaller and comparatively less distinct than those resulting from infection with SFG organisms (Hackstadt, 1996; Policastro et al., 1996).

\section{Dynamics of NF- $\kappa$ B activation in ECs infected with $R$. conorii and $R$. typhi}

With the aim of defining potential similarities and/or differences in the host-cell response(s) to infection with spotted fever versus typhus rickettsiae, we initially focused on characteristic features and kinetics of infection-induced activation of NF- $\kappa \mathrm{B}$. Nuclear protein extracts isolated from ECs infected for varying lengths of time ranging from 3 to $24 \mathrm{~h}$ were subjected to an EMSA. The data clearly suggested that $R$. conorii infection triggered a biphasic pattern of NF$\kappa \mathrm{B}$ activation, with an early increase in DNA-binding activity evident at 3 and $7 \mathrm{~h}$, followed by a decrease towards the basal level at $14 \mathrm{~h}$ and another later peak of enhanced NF- $\kappa$ B binding activity at $24 \mathrm{~h}$ post-infection (p.i.) (Fig. 1a, c). The mean increase in R. conorii-infected ECs at $3 \mathrm{~h}$, as measured by densitometric scanning and image analysis, was about fivefold in comparison with the basal level in uninfected control cells. The secondary peak of NF- $\kappa$ B activation at $24 \mathrm{~h}$ was comparatively less intense than the earlier peak of activation. R. typhi infection, on the other hand, caused significant activation of NF- $\kappa \mathrm{B}$ at $3 \mathrm{~h}$ and this response was sustained up to $7 \mathrm{~h}$ p.i., followed by a decline to the baseline level. Interestingly, no changes in the DNAbinding activity pattern of NF- $\kappa$ B were apparent at the later time points of 21 and $24 \mathrm{~h}$ (Fig. 1b, c). The intensity of the NF- $\kappa \mathrm{B}$ activation in ECs infected with $R$. typhi for $3 \mathrm{~h}$ was 
(a)

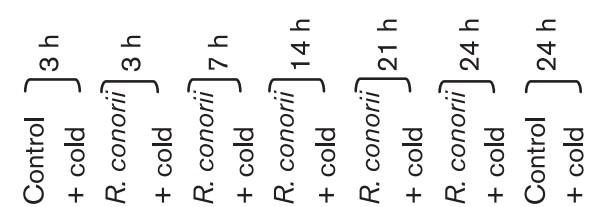

(b)

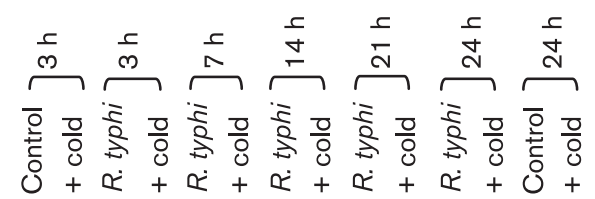

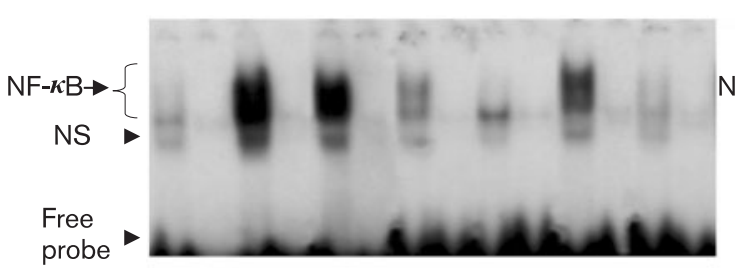

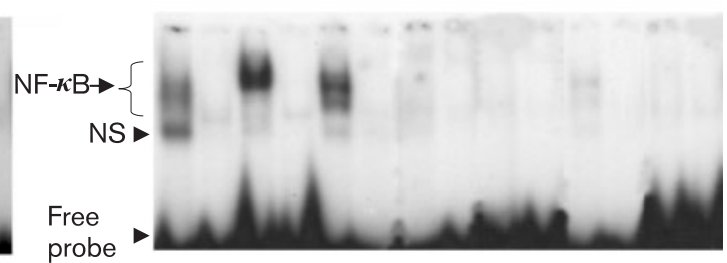

(c)

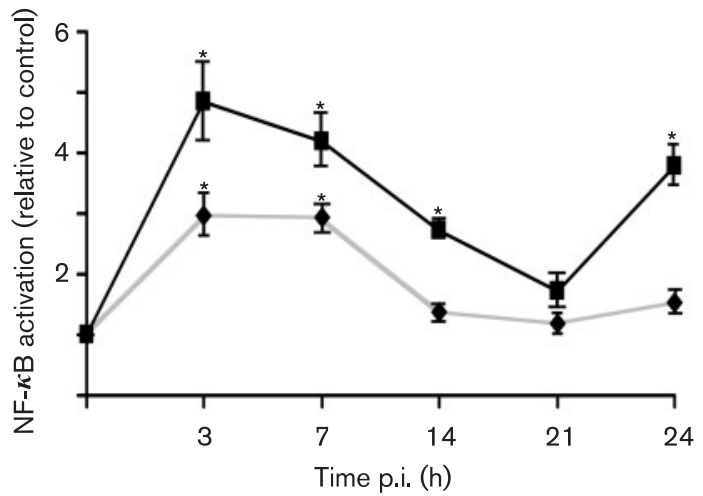

Fig. 1. Kinetics of $R$. conorii- and $R$. typhi-induced NF- $\kappa$ B activation. Endothelial cells were incubated with culture medium alone (control) or infected with approximately $6 \times 10^{4}$ p.f.u. $R$. conorii (a) or $R$. typhi (b) (cm ${ }^{2}$ culture surface area) $)^{-1}$ for $3,7,14$, 21 and 24 h. Nuclear protein extracts were processed for EMSA using [ $\gamma^{-32}$ P]ATP-labelled consensus NF- $\kappa$ B-binding oligonucleotide and the specificity of the gel-shifted NF- $\kappa$ B complex was ascertained by inclusion of a 100-fold molar excess of unlabelled probe (+ cold) during DNA-protein binding. The relative position of shifted NF- $\kappa$ B dimers, a non-specific band (NS) and free probe are indicated. The quantitative densitometric analysis of the NF- $\kappa \mathrm{B}$ activation response during $R$. conorii ( $\boldsymbol{\square})$ and $R$. typhi ( infection of cultured ECs is shown in (c) as a function of time. The values are presented as mean $\pm \mathrm{SEM}$ of a minimum of three independent experiments. For comparison, the basal levels in uninfected cells (control) were assigned a value of 1 . *Statistically significant changes $(P \leqslant 0.05)$ in $R$. conorii- and $R$. typhi-infected cells compared with the baseline values in the corresponding samples from uninfected ECs.

about threefold higher than uninfected controls, but was noticeably lower than that for $R$. conorii infection.

To identify the component NF- $\kappa \mathrm{B}$ subunits present in the activated complex, antibody supershift analysis was performed using specific antibodies against p50, p65 (Rel A) and c-Rel. Uninfected ECs displayed minimal levels of supershifted complexes reactive with antibody against p50 (Fig. 2a). In nuclear extracts prepared from $R$. conorii- and $R$. typhi-infected ECs, the presence of anti-p50 and antip65 antibodies in the reaction mixture resulted in the formation of slower-migrating supershifted complexes and concomitant loss of intensity of the original NF- $\kappa \mathrm{B}$ complex, which migrated to a lower position on the gel (Fig. 2). Activated NF- $\kappa$ B complexes in R. conorii-infected as well as $R$. typhi-infected ECs at $3 \mathrm{~h}$ had identical subunit composition and were found to consist primarily of p65p50 heterodimers and p50 homodimers (Fig. 2b, c). Consistent with our earlier observations with $R$. rickettsii
(Sporn et al., 1997), ECs infected with $R$. conorii and $R$. typhi did not activate any c-Rel-containing dimers (Fig. 2). Similar analysis of the later peak of the NF- $\kappa$ B complex in $R$. conorii-infected ECs further suggested that it comprised heterodimers made up of p65 and p50 or homodimers of p50 and thus had the same composition as the early peak of activation (data not shown).

I $\kappa \mathrm{B}$ (inhibitor of $\kappa \mathrm{B}$ ) proteins play an important role in the canonical pathway of activation by retaining $\mathrm{NF}-\kappa \mathrm{B}$ in the cytoplasm as an inactive $\mathrm{NF}-\kappa \mathrm{B}-\mathrm{I} \kappa \mathrm{B}$ complex. Among the known $\mathrm{I} \kappa \mathrm{B}$ proteins, $\mathrm{I} \kappa \mathrm{B} \alpha$ is abundantly expressed in a variety of mammalian cells (Viatour et al., 2005). Because a prerequisite signalling step for $\mathrm{I} \kappa \mathrm{B} \alpha$ degradation by the proteasome and release of active NF- $\kappa \mathrm{B}$ involves the phosphorylation of specific carboxyl-terminus serine residues (Ser32 and Ser36), we also investigated the phosphorylation status of $\mathrm{I} \kappa \mathrm{B} \alpha$ after EC infection with $R$. conorii and $R$. typhi by Western blotting with 
(a)

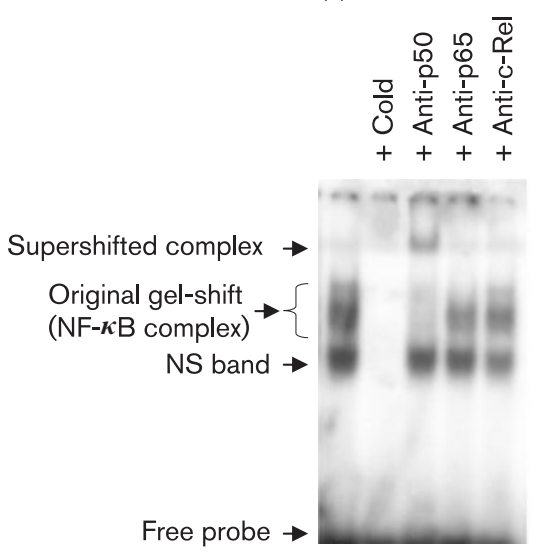

(b)

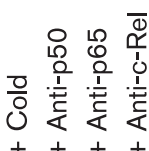

(c)

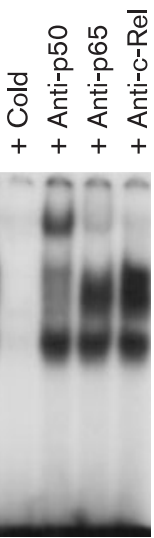

Fig. 2. Antibody supershift analysis of activated NF- $\kappa \mathrm{B}$ species in $R$. conorii- and $R$. typhi-infected ECs. Nuclear extracts from host ECs incubated with culture medium alone (a) or infected for $3 \mathrm{~h}$ with $R$. conorii (b) or R. typhi (c) were subjected to gel supershift analysis. Specific antibodies against the NF- $\kappa \mathrm{B}$ subunits p50, p65 and c-Rel were incubated with nuclear proteins at $37{ }^{\circ} \mathrm{C}$ for 20 min prior to conducting DNA-protein binding reactions with isotopically labelled NF- $\kappa \mathrm{B}$ oligonucleotide probe. The relative positions of supershifted complexes, gel-shifted complexes in the absence of antibody in the assay mix, a non-specific (NS) complex and free excess probe are indicated.
anti-phospho-I $\kappa \mathrm{B} \alpha$ (Ser32-specific) antibody capable of detecting endogenous levels of $\mathrm{I} \kappa \mathrm{B} \alpha$ phosphorylated at Ser32. EC infection for $60 \mathrm{~min}$ with either Rickettsia species led to markedly increased phosphorylation of $\mathrm{I} \kappa \mathrm{B} \alpha$. The abundance of phosphorylated $\mathrm{I} \kappa \mathrm{B} \alpha$ in $R$. conorii- and $R$. typhi-infected cells was significantly increased at 1.5, 3 and $6 \mathrm{~h}$ (Fig. 3), apparently coinciding with the kinetics of NF$\kappa \mathrm{B}$ activation. Although the intensity of $\mathrm{I} \kappa \mathrm{B} \alpha$ phosphorylation in $R$. conorii-infected host cells was higher than in those infected with $R$. typhi, the differences at all tested time points were statistically insignificant.

The transcription factor NF- $\kappa \mathrm{B}$, which plays a critical role in the regulation of cellular immune and inflammatory responses (Xiao \& Ghosh, 2005; Liu \& Malik, 2006), is composed of homo- and heterodimers of the Rel family of proteins. Our data for $R$. conorii and $R$. typhi in this study and those reported previously for $R$. rickettsii (Sporn et al., 1997; Sahni et al., 1998) demonstrated that the activated complexes are mainly composed of two major NF- $\kappa \mathrm{B}$ subunits, p65 (Rel A) and p50 (NF- $\kappa \mathrm{B} 1)$, in the form of either p65-p50 heterodimers or homodimers made up of p50. As would be expected based on their phylogenetic proximity and the hallmark characteristics of disease pathogenesis in humans, the activation profile of NF- $\kappa \mathrm{B}$ during $R$. conorii infection of ECs exhibited a biphasic response strikingly similar to that for $R$. rickettsii. Infection of host ECs with $R$. typhi, however, produced only a transient increase in the DNA-binding activity of NF- $\kappa \mathrm{B}$. Comparative analysis with a representative species for each major subgroup of Rickettsia species thus suggests that, whilst activation of p65- and p50-containing NF- $\kappa \mathrm{B}$ components most likely represents a common host-cell transcriptional response to infection, remarkable differences in the activation profile also exist. Using measurement of expression of $\mathrm{I} \kappa \mathrm{B} \alpha$, a protein involved in the autoregulatory shut-off mechanism to inhibit sustained NF- $\kappa$ B activation, Radulovic et al. (2002) provided indirect evidence for increased NF- $\kappa$ B activity in P388D1 cells and peritoneal macrophages infected in vitro with $R$. typhi and $R$. akari. Based on the differential expression of cytokines, this study further advocates the possibility of biological differences among these closely related intracellular pathogens.

Increased phosphorylation of $\mathrm{I} \kappa \mathrm{B} \alpha$, an essential step for its subsequent ubiquitination and degradation by the

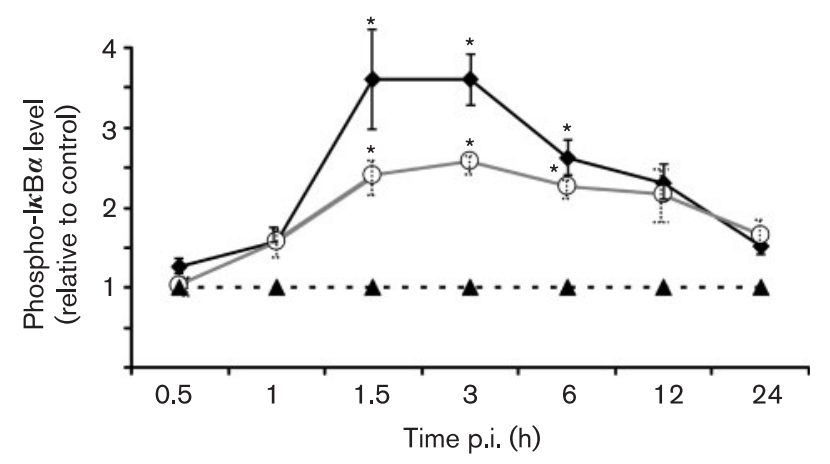

Fig. 3. Comparative kinetic analysis of $\mathrm{l}_{\kappa} \mathrm{B} \alpha$ phosphorylation during $R$. conorii and $R$. typhi infection. Total protein lysates prepared from ECs that were left uninfected (control) $(\boldsymbol{A})$ or were infected for the indicated times with $R$. conorii $(\boldsymbol{\nabla})$ or $R$. typhi $(\bigcirc)$ were subjected to SDS-PAGE, followed by blotting onto nitrocellulose membrane and probing with phosphorylation-statespecific $I_{\kappa} \mathrm{B} \alpha$ (Ser32) antibody followed by anti- $\alpha$-tubulin antibody. The films with optimum exposures were scanned and individual bands for different experimental conditions were quantified densitometrically. For all experiments, the level of $\mathrm{l} \kappa \mathrm{B} \alpha$ phosphorylation in uninfected cells was assigned a value of one to determine the relative fold induction compared with the baseline level. The data are presented as the arithmetic mean \pm SEM of at least three independent observations. *Statistically significant changes $(P \leqslant 0.05)$ in $R$. conorii- and $R$. typhi-infected cells compared with the baseline values in the corresponding samples from uninfected ECs. 
proteasome in $R$. conorii- as well as $R$. typhi-infected cells early during the infection further indicates that host-cell invasion by rickettsiae generates some common upstream signals that culminate in the activation of the $\mathrm{I} \kappa \mathrm{B}$ kinase complex, degradation of sequestering protein $\mathrm{I} \kappa \mathrm{B} \alpha$ and ultimately in the nuclear translocation of NF- $\kappa \mathrm{B}$. Our earlier finding that $R$. rickettsii-induced synthesis and secretion of IL- $1 \alpha$ contributes, in part, to the late phase of the NF- $\kappa \mathrm{B}$ response may also hold true for cells infected with $R$. conorii, as it has been shown that in vitro infection with both $R$. rickettsii and $R$. conorii induces expression and synthesis of IL- $1 \alpha$ but not IL- $1 \beta$, and that most of it remains cell associated (Kaplanski et al., 1995; Sporn \& Marder, 1996). Although R. typhi-infected macrophages reportedly produce and secrete IL- $1 \beta$ (Radulovic et al., 2002), whether or not infected ECs respond in a similar fashion and the possibility of its role in determining other host-cell responses need to be investigated in further detail.

\section{Dynamics of p38 MAPK activation in ECs infected with $R$. conorii and $R$. typhi}

MAPK pathways, as well as NF- $\kappa \mathrm{B}$, govern cellular inflammatory and immune responses by regulating proinflammatory ILs and other mediators such as cyclooxygenase-2, intercellular adhesion molecule-1, inducible nitric oxide synthase and haem oxygenase-1. The p38 MAPK subfamily is known to mediate stress responses, such as apoptosis and immune signalling. Indeed, NF- $\kappa \mathrm{B}$ and p 38 MAPK are both pivotal to the cytokine formation induced by $R$. rickettsii (Clifton et al., 2005; Rydkina et al., 2005). As all MAPKs are activated following dual phosphorylation of the threonine-X-tyrosine motif, where $\mathrm{X}$ is glycine in $\mathrm{p} 38$ kinases, we carried out further evaluation of the abilities of $R$. conorii and R. typhi to trigger p38 MAPK activity in ECs by determining the phosphorylation status of p38 kinases. Our results demonstrated that, whilst both species of rickettsiae were able to stimulate phosphorylation of p38 MAPK, notable differences in the intensity but not the kinetics of its activation were also evident. Infection of ECs with both SF and TG species resulted in p38 MAPK activation, as indicated by higher intracellular levels of phospho-p38. In $R$. conorii ECs, p38 phosphorylation started to increase at $0.5-1 \mathrm{~h}$ and peaked at $3 \mathrm{~h}$. The decline towards the baseline control level at $12 \mathrm{~h}$ was followed by a slight but significant increase at $24 \mathrm{~h}$ p.i. (Fig. 4a). The accumulation of phospho-p38 in response to $R$. typhi also displayed a progressive increase starting at $0.5 \mathrm{~h}$, with the maximum level at $6 \mathrm{~h}$ (Fig. $4 \mathrm{~b}$ ). For R. conorii, the maximal activation relative to the control at $3 \mathrm{~h}$ was about $3.2 \pm 0.6$ fold, whereas activation of $\mathrm{p} 38$ in ECs infected with $R$. typhi was considerably weaker, as suggested by a maximum increase of $1.9 \pm 0.2$-fold in relation to the untreated control (Fig. 4c).

Available clinical data and experimental animal models of infection indicate that vascular inflammation is of major importance in the pathogenesis of rickettsial diseases
(Olano, 2005; Sahni, 2007; Valbuena \& Walker, 2006). In general, a variety of NF- $\kappa$ B-inducing inflammatory stimuli including TNF and LPS also activate upstream signalling pathways involving tyrosine kinase and p38, p42/44 (ERK) and JNK (stress-activated protein kinase) MAPKs, which can, in turn, further regulate the activation of transcription factors (Ghosh, 1999). Previous work from our laboratory has shown that increased nuclear translocation and the DNA-binding activity of NF- $\kappa \mathrm{B}$ in $R$. rickettsii-infected ECs are not mediated by p38 kinase activation (Rydkina et al., 2005). Our comparative studies aimed at identifying early intracellular signalling steps during $R$. conorii and $R$. typhi infection demonstrate that although p38 phosphorylation occurs as early as 30-60 min after rickettsial contact with ECs and follows an apparently similar kinetic pattern of activation, $R$. conorii is able to stimulate this signalling mechanism to a higher intensity than $R$. typhi. Whether or not such differences in the elicitation of inflammatory networks correlate with the extent of vascular injury or disease severity in established in vivo models of infection remains to be determined. Currently in progress, additional studies to decipher the potential roles of cell-wall components and other rickettsial proteins such as LPS, outer-membrane proteins and heat-shock proteins or recently identified putative virulence factors such as phospholipase D in the initiation and maintenance of signalling pathways should reveal further important information regarding the specifics of host-cell interactions with SFG and TG rickettsiae.

\section{Effect of $R$. conorii and $R$. typhi infection on chemokine gene expression and secretion}

In subsequent comparative studies, transcriptional expression and release of regulatory chemokines by ECs in response to infection with $R$. conorii and $R$. typhi were also examined. The relative mRNA expression of selected chemokine genes was analysed using RNA from ECs infected for varying lengths of time and semi-quantitative multiplex PCR. At all times p.i., both $R$. conorii- and $R$. typhi-infected ECs displayed marked upregulation of IL-8 and MCP-1 expression compared with uninfected cells (Fig. 5a). An increase in the number of amplification cycles to attain optimal generation of PCR products for other chemokines, which were expressed at levels considerably lower than IL-8 and MCP-1, also showed significant increases in the expression of IP-10, MCP-2 and Rantes, indicating a pattern of inflammatory/immune response similar to that for $R$. rickettsii (Rydkina et al., 2005).

The levels of IL- 8 and MCP-1 in the culture supernatants of infected cells were determined by ELISA measurements. The secretion patterns for these chemokines for $R$. conoriiand $R$. typhi-infected ECs and simultaneous controls are shown in Fig. 5(b, c), respectively. Whilst uninfected cells secreted low but detectable amounts of IL-8 (1.4-1.9 ng $\mathrm{ml}^{-1}$ at $3 \mathrm{~h}$ and $3.3-5.2 \mathrm{ng} \mathrm{ml}^{-1}$ at $\left.24 \mathrm{~h}\right)$ and MCP-1 (1.8$2.3 \mathrm{ng} \mathrm{ml}^{-1}$ at $3 \mathrm{~h}$ and $5.0-6.3 \mathrm{ng} \mathrm{ml}^{-1}$ at $24 \mathrm{~h}$ ) into the 

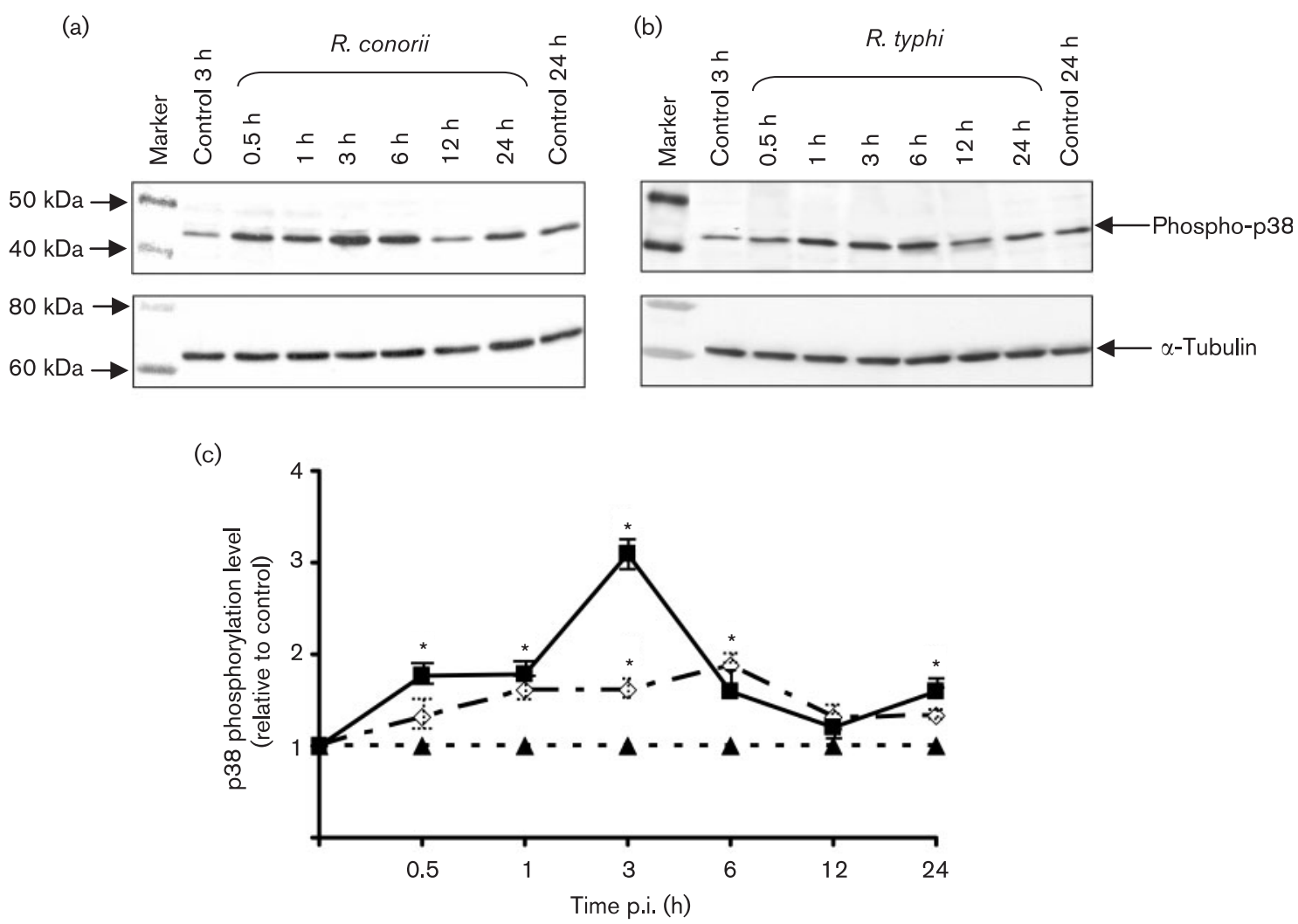

Fig. 4. Activation of the p38 MAPK module in $R$. conorii- and $R$. typhi-infected ECs at different times after infection. Total protein lysates from cells infected with $R$. conorii or $R$. typhi for different durations ranging from 30 min to $24 \mathrm{~h}$ and corresponding uninfected controls ( 3 and $24 \mathrm{~h}$ ) were analysed by Western blotting to determine the level of phosphorylated p38 kinase. Blots were first probed with a phosphorylation-state-specific p38 (Thr180/Tyr182) antibody, followed by an anti- $\alpha$ tubulin antibody to normalize for variations in protein loading among the different samples. Results of a representative blot each for R. conorii (a) and R. typhi (b) are shown. The positions of biotinylated molecular mass markers detected with an anti-biotin antibody are also indicated. The detailed densitometric analysis of changes in phospho-p38 level as the mean fold induction over the basal level \pm SEM $(n \geqslant 3)$ is shown in (c). For these calculations, the mean baseline level of p38 phosphorylation in uninfected ECs (control) was assigned a value of one. *Statistically significant changes $(P \leqslant 0.05)$ in $R$. conorii- and $R$. typhiinfected cells compared with the baseline values in the corresponding samples from uninfected ECs. $\diamond, R$. typhi;,$R$. conorii;

$\boldsymbol{\Delta}$, control.

culture medium, infection for $3 \mathrm{~h}$ with either $R$. conorii or $R$. typhi resulted in enhanced secretion of both chemokines compared with the corresponding baseline secretion by uninfected controls (Fig. 5b, c). Although the magnitude of increased release of IL- 8 and MCP-1 at 3 h p.i. with $R$. conorii and $R$. typhi was nearly identical, noticeable differences in the secretion patterns of both chemokines were also apparent at later times. As shown in Fig. 5, $R$. conorii infection triggered a time-dependent pattern of increased IL-8/MCP-1 secretion with significant differences compared with uninfected controls starting from $3 \mathrm{~h}$ p.i. The mean concentration of IL- 8 in culture supernatants of R. conorii-infected cells increased from $6.5 \mathrm{ng} \mathrm{ml}^{-1}$ at $3 \mathrm{~h}$ to $58.3 \mathrm{ng} \mathrm{ml}^{-1}$ at $24 \mathrm{~h}$ and that for MCP-1 ranged from $12.2 \mathrm{ng} \mathrm{ml}^{-1}$ at $3 \mathrm{~h}$ to $82.2 \mathrm{ng} \mathrm{ml}^{-1}$ later in the infection. Accordingly, the mean fold induction in relation to corresponding controls was about 5 -fold and 6 -fold at $3 \mathrm{~h}$ and 15 -fold at 24 h. R. typhi infection, however, produced only an early response with a mean value of $6.8 \mathrm{ng} \mathrm{ml}^{-1}$ for IL- 8 and $13.8 \mathrm{ng} \mathrm{ml}^{-1}$ for MCP-1 at $3 \mathrm{~h}$ p.i. Interestingly, there was no further increase in IL-8 secretion by $R$. typhiinfected ECs up to $24 \mathrm{~h}$ after infection and the mean increase in comparison with uninfected control was 3.3fold and 3.5-fold at 3 and $24 \mathrm{~h}$, respectively. MCP-1 secretion by $R$. typhi-infected ECs was also stable from 3 to $12 \mathrm{~h}$ p.i., exceeding the control level by about 5- to 6-fold, followed by a further increase to about 9.6-fold (mean of $39.1 \mathrm{ng} \mathrm{ml}^{-1}$ ) above the basal secretion level at $24 \mathrm{~h}$.

The interpretation of findings from the analysis of chemokine expression illustrates that the predominantly intracytoplasmic $R$. typhi and the highly motile $R$. conorii direct fundamentally similar responses in host cells. As shown, the expression of both IL-8 and MCP-1 was clearly upregulated consequent to endothelial infection with both species of pathogenic rickettsiae used in our experiments. 


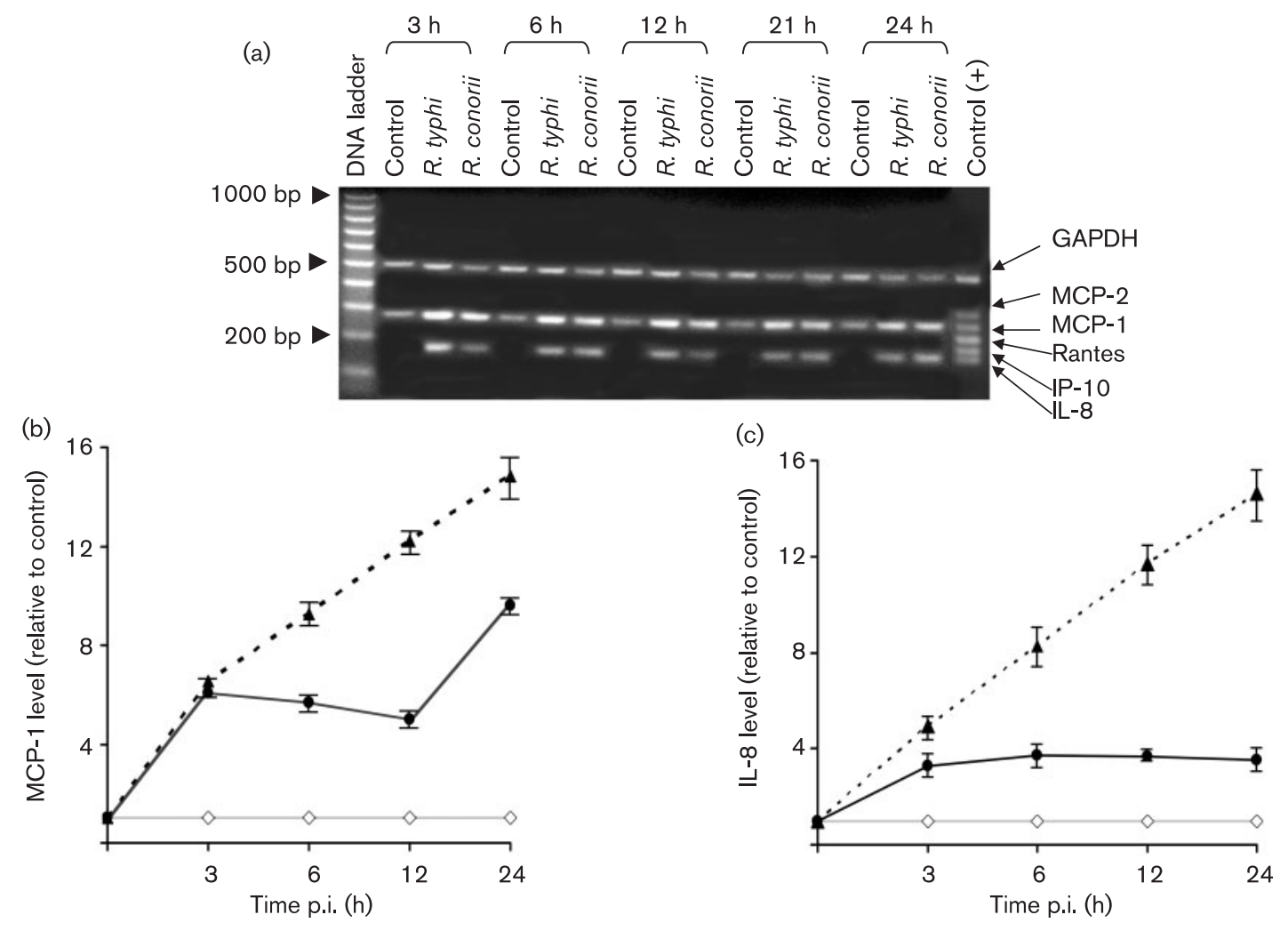

Fig. 5. mRNA expression and secretion of IL-8 and MCP-1 during $R$. conorii and $R$. typhi infection. RNA samples and culture supernatants from uninfected ECs (control) or ECs infected with $R$. conorii or $R$. typhi for the indicated periods of time were subjected to multiplex RT-PCR for analysis of chemokine expression (a) and ELISA to measure the level of MCP-1 (b) and IL-8 (c). The RNA sample $(4 \mu \mathrm{g})$ for each experimental condition was reverse transcribed using SuperScript II RNase $\mathrm{H}^{-}$reverse transcriptase and the PCRs for each transcript tested were performed in the linear range using the housekeeping gene GAPDH as a reference control. The PCR products obtained from experimental samples and a positive control provided by the assay manufacturer were resolved simultaneously on a $2 \%$ agarose gel and visualized by ethidium bromide staining. The sizes for the IL-8, IP-10, Rantes, MCP-1, MCP-2 and GAPDH amplicons were 173, 199, 233, 277, 330 and 500 bp, respectively. All assays were performed with at least three separate sets of RNA samples with similar results, of which a representative gel for IL8 and MCP-1 is shown. To compare the secretion patterns of IL- 8 and MCP-1, the levels of IL- 8 and MCP- 1 secreted by uninfected ECs were assigned a value of one and the fold induction over the baseline level was then determined. Data derived from a minimum of three independent experiments are presented as the mean \pm SEM. $\bullet, R$. typhi; $\boldsymbol{\Delta}, R$. conorii; $\diamond$, control.

IL-8, a CXC chemokine also known as CXCL8, not only promotes the migration of leukocytes (including basophils, monocytes, $\mathrm{T}$ and $\mathrm{B}$ lymphocytes and neutrophils) to the sites of infection, but also participates in inflammationinduced angiogenesis (Kofler et al., 2005). The involvement of neutrophils in rickettsial vasculitis is, in general, considered to be relatively rare (Elghetany \& Walker, 1999), but intriguing clinical evidence suggesting infiltration of large mononuclear cells and neutrophils in the hepatic portal triad in humans has also been reported (Adams \& Walker, 1981). More recent findings also suggest neutrophil recruitment as a prominent component of the host's cellular immune response in patients with Astrakhan fever (a disease similar to Mediterranean spotted fever and endemic in the Astrakhan region of Russia; Kasimova et al., 2002; Fournier et al., 2003) and African tick-bite fever (Lepidi et al., 2006). As severe leukocytosis accompanied by toxic granulation of neutrophils in dogs diagnosed with Rocky Mountain spotted fever has been shown to occur (Gasser et al., 2001), it is also possible that IL-8 may be involved indirectly in mediating T-cell accumulation at sites of vascular inflammation by inducing neutrophil degranulation and release of other potent chemoattractants (Taub et al., 1996). Furthermore, although IL-8 is recognized as a major neutrophil-activating factor, $\mathrm{T}$ lymphocytes exhibit even higher sensitivity to its chemotactic activity (Larsen et al., 1989), most likely contributing to lymphocytic vasculitis associated with rickettsial diseases (Kaplanski et al., 1995; Carlson \& Chen, 2007). However, it should also be kept in mind that, contrary to ECs cultured in vitro under static conditions, the vascular endothelium in vivo is constantly exposed to fluid mechanical forces and, accordingly, soluble chemokine gradients are highly unlikely to establish at the luminal endothelial surface, 
where chemokines would be washed away due to blood flow. In this regard, in vitro findings of $R$. rickettsii-induced expression of E-selectin on the host-cell surface and IL-8 secretion by ECs are supported by increased concentrations of IL-8 and soluble E-selectin in the circulating blood of a patient with fulminant Rocky Mountain spotted fever (Sporn et al., 1993; Sessler et al., 1995; Sessler \& Fowler, 1996; Clifton et al., 2005). Similar systemic inflammatory responses are also evident during African tick-bite fever (Jensenius et al., 2003), but a prospective study of 500 patients with confirmed diagnosis of Mediterranean spotted fever revealed undetectable blood levels of IL-8 and IL-1 at all stages of infection (Vitale et al., 2001), although cultured ECs infected with $R$. conorii secrete IL-8 via enhanced synthesis and autocrine effects of a cellassociated form of IL-1 $\alpha$ (Kaplanski et al., 1995). Taken together, these observations highlight the need for vigorous efforts directed at comprehensive examination of potential correlations between endothelial responses in vitro and the onset/establishment of hypercytokinemia in vivo.

The CC chemokine MCP-1 is known to contribute to the chemotaxis of chemokine receptor 3-expressing inflammatory cells including macrophages and lymphocytes. In addition, the transcriptional expression profile of ECs infected with $R$. typhi and R. conorii was nearly identical to that triggered by $R$. rickettsii, as evidenced by significantly increased expression of IL-8, MCP-1, Rantes, MCP-2 and IP-10. Earlier studies have documented that the response of macrophages to $R$. typhi primarily involves significantly increased secretion of TNF- $\alpha$, IL- $1 \beta$ and IL-6 (Radulovic et al., 2002). Although together these observations suggest the likelihood of unique interactions between invasive rickettsiae and different host-cell types, it is also important to consider that the SDG and TG rickettsiae have been shown to stimulate T-cell-mediated reciprocal crossimmunity in the immunocompetent host (Valbuena et al., 2004). The pattern of MCP-1 production and release by host cells harbouring $R$. typhi coincided with an initial response to invasion, which was followed by another spike in secretion corresponding to the replication of intracellular organisms. In contrast, IL-8 secretion by $R$. typhiinfected ECs did not increase with the progress of infection and remained stationary at levels seen at $3 \mathrm{~h}$ p.i., suggesting the possible involvement of post-transcriptional regulatory mechanisms or the potential lack of interactions with Weibel-Palade bodies. Identified originally as the intracellular storage site for von Willebrand factor in ECs, recent evidence confirming the presence of physiologically important mediators such as angiopoietin, endothelin, eotaxin and IL-8 in Weibel-Palade bodies, and release of these components after vascular perturbation has pointed towards a critical role for this subcellular organelle in inflammation, haemostasis and regulation of vessel tone (Rondaij et al., 2006). Therefore, an important aspect that warrants further detailed characterization is whether or not infection with TG rickettsiae leads to interactions with and release of markers associated with Weibel-Palade bodies, a phenomenon previously described in the literature as enhanced release of von Willebrand factor during infection with the SFG species $R$. rickettsii and $R$. conorii (Sporn et al., 1991; Teysseire et al., 1992). As an essential corollary, the highly complex topology of vascular endothelium and evidence for a large array of unique, site-specific phenotypes of ECs from different anatomical locations in vivo, a concept collectively termed EC heterogeneity (Aird, 2006), should also be evaluated carefully. This acquires additional physiological significance when one considers that the vascular sequelae of human rickettsioses are primarily associated with damage to microcirculatory networks, most likely due to preferential targeting of small and medium-sized vessels.

\section{Conclusions}

The characterization of host-cell inflammatory reactions to infection with different types of bacteria at the molecular level has revealed not only some common responses but also distinct patterns of cytokine gene expression, suggesting the existence of biological complexities that determine the host response to different pathogens (Feezor et al., 2003). Although pathological consequences of human infections with well-established pathogenic Rickettsia species belonging to the SFG and TG implicate disseminated infection of the endothelial cell lining of various organ systems leading to vascular damage, inflammation and permeability changes (Olano, 2005; Sahni, 2007; Valbuena \& Walker, 2006), the specifics of interactions between different species of pathogenic rickettsiae and their preferred host cells remain poorly understood. Whereas sequencing and annotation of entire genomes of $R$. prowazekii (Andersson et al., 1998), R. conorii (Ogata et al., 2001) and R. typhi (McLeod et al., 2004), and comparative genomics indicate a number of similar features such as small overall genomic size, low GC content and the presence of a total of 776 common genes, remarkable differences exemplified by the presence of 13 , 24 and 560 unique genes in $R$. prowazekii, $R$. typhi and $R$. conorii, respectively, are also evident (Walker \& Yu, 2005). In addition, there also exist significant differences in the repertoire of outer-surface protein antigens and intracellular actin-based motility patterns of SFG versus TG rickettsiae (Hackstadt, 1996; Heinzen, 2003; Olano, 2005). Using an in vitro model system that exploits infection of cultured human ECs with viable rickettsiae and that has been thoroughly characterized by our and other laboratories to investigate host cell- $R$. rickettsii interactions (Eremeeva et al., 2000), we have compared the transcriptional activation and chemokine gene expression after comparable levels of infection with $R$. conorii and $R$. typhi. The results suggest that, similar to signalling mechanisms activated by $R$. rickettsii, cells infected with $R$. conorii also display a biphasic pattern of NF- $\kappa$ B activation, increased phosphorylation of $\mathrm{p} 38$ MAPK reflecting activation of this inflammatory kinase module and a robust increase in the expression and secretion of the important chemokines IL-8 and MCP-1. R. typhi infection of host ECs, on the other 
hand, triggers similar cell-signalling events, but the magnitude and/or kinetics of the resultant responses reflect significant variations in comparison with those seen with $R$. conorii. The mechanisms underlying the development of proinflammatory effects and the extent to which they are activated may ultimately determine the degree of vascular dysfunction and damage in the infected host and most likely contribute to the pathogenesis of human rickettsial diseases. Accordingly, selective inhibitors of these or other pathways could be exploited as effective therapeutic modalities to combat the detrimental pathological effects of pathogenic Rickettsia species.

\section{ACKNOWLEDGEMENTS}

We gratefully acknowledge the financial support from the National Institute of Allergy and Infectious Diseases, NIH, through research grant AI 040689, and the excellent technical assistance provided by Loel Turpin and Semion Kiriakidi.

\section{REFERENCES}

Adams, J. S. \& Walker, D. H. (1981). The liver in Rocky Mountain spotted fever. Am J Clin Pathol 75, 156-161.

Aird, W. C. (2006). Mechanisms of endothelial cell heterogeneity in health and disease. Circ Res 98, 159-162.

Andersson, S. G., Zomorodipour, A., Andersson, J. O., SicheritzPonten, T., Alsmark, U. C., Podowski, R. M., Naslund, A. K., Eriksson, A. S., Winkler, H. H. \& Kurland, C. G. (1998). The genome sequence of Rickettsia prowazekii and the origin of mitochondria. Nature 396, 133-140.

Audia, J. P. \& Winkler, H. H. (2006). Study of the five Rickettsia prowazekii proteins annotated as ATP/ADP translocases (Tlc): only Tlc1 transports ATP/ADP, while Tlc4 and Tlc5 transport other ribonucleotides. J Bacteriol 188, 6261-6268.

Boyd, A. S. (1997). Rickettsialpox. Dermatol Clin 15, 313-318.

Carlson, J. A. \& Chen, K. R. (2007). Cutaneous vasculitis update: neutrophilic muscular vessel and eosinophilic, granulomatous, and lymphocytic vasculitis syndromes. Am J Dermatopathol 29, 32-43.

Clifton, D. R., Rydkina, E., Huyck, H., Pryhuber, G., Freeman, R. S., Silverman, D. J. \& Sahni, S. K. (2005). Expression and secretion of chemotactic cytokines IL-8 and MCP-1 by human endothelial cells after Rickettsia rickettsii infection: regulation by nuclear transcription factor NF- $\kappa$ B. Int J Med Microbiol 295, 267-278.

Dignat-George, F., Teysseire, N., Mutin, M., Bardin, N., Lesaule, G., Raoult, D. \& Sampol, J. (1997). Rickettsia conorii infection enhances vascular cell adhesion molecule-1- and intercellular adhesion molecule-1-dependent mononuclear cell adherence to endothelial cells. J Infect Dis 175, 1142-1152.

Elghetany, M. T. \& Walker, D. H. (1999). Hemostatic changes in Rocky Mountain spotted fever and Mediterranean spotted fever. Am J Clin Pathol 112, 159-168.

Eremeeva, M. E., Dasch, G. A. \& Silverman, D. J. (2000). Interaction of rickettsiae with eukaryotic cells. Adhesion, entry, intracellular growth, and host cell responses. Subcell Biochem 33, 479-516.

Feezor, R. J., Oberholzer, C., Baker, H. V., Novick, D., Rubinstein, M., Moldawer, L. L., Pribble, J., Souza, S., Dinarello, C. A. \& other authors (2003). Molecular characterization of the acute inflammatory response to infections with Gram-negative versus Gram-positive bacteria. Infect Immun 71, 5803-5813.
Fournier, P. E., Durand, J. P., Rolain, J. M., Camicas, J. L., Tolou, H. \& Raoult, D. (2003). Detection of Astrakhan fever rickettsia from ticks in Kosovo. Ann N Y Acad Sci 990, 158-161.

Gasser, A. M., Birkenheuer, A. J. \& Breitschwerdt, E. B. (2001). Canine Rocky Mountain spotted fever: a retrospective study of 30 cases. J Am Anim Hosp Assoc 37, 41-48.

Ghosh, S. (1999). Regulation of inducible gene expression by the transcription factor NF- $\kappa$ B. Immunol Res 19, 183-189.

Gouin, E., Gantelet, H., Egile, C., Lasa, I., Ohayon, H., Villiers, V., Gounon, P., Sansonetti, P. J. \& Cossart, P. (1999). A comparative study of the actin-based motilities of the pathogenic bacteria Listeria monocytogenes, Shigella flexneri, and Rickettsia conorii. J Cell Sci 112, 1697-1708.

Gouin, E., Egile, C., Dehoux, P., Villiers, V., Adams, J., Gertler, F., Li, R. \& Cossart, P. (2004). The RickA protein of Rickettsia conorii activates the Arp2/3 complex. Nature 427, 457-461.

Hackstadt, T. (1996). The biology of rickettsiae. Infect Agents Dis 5, 127-143.

Hackstadt, T. (1998). The diverse habitats of obligate intracellular parasites. Curr Opin Microbiol 1, 82-87.

Heinzen, R. A. (2003). Rickettsial actin-based motility: behavior and involvement of cytoskeletal regulators. Ann N Y Acad Sci 990, 535-547.

Heinzen, R. A., Grieshaber, S. S., Van Kirk, L. S. \& Devin, C. J. (1999). Dynamics of actin-based movement by Rickettsia rickettsii in Vero cells. Infect Immun 67, 4201-4207.

Jeng, R. L., Goley, E. D., D’Alessio, J. A., Chaga, O. Y., Svitkina, T. M., Borisy, G. G., Heinzen, R. A. \& Welch, M. D. (2004). A Rickettsia WASP-like protein activates the Arp2/3 complex and mediates actinbased motility. Cell Microbiol 6, 761-769.

Jensenius, M., Ueland, T., Fournier, P. E., Brosstad, F., Stylianou, E., Vene, S., Myrvang, B., Raoult, D. \& Aukrust, P. (2003). Systemic inflammatory responses in African tick-bite fever. J Infect Dis 187, 1332-1336.

Kaplanski, G., Teysseire, N., Farnarier, C., Kaplanski, S., Lissitzky, J. C., Durand, J. M., Soubeyrand, J., Dinarello, C. A. \& Bongrand, P. (1995). IL-6 and IL-8 production from cultured human endothelial cells stimulated by infection with Rickettsia conorii via a cellassociated IL-1 $\alpha$-dependent pathway. J Clin Invest 96, 2839-2844.

Kasimova, N. B., Kulikova, I. N., Karpenko, S. F., Burkin, V. S., Altukhov, S. A., Arshba, T. E. \& Airapetova, G. S. (2002). Status of cellular immunity in patients with Astrakhan fever. Ter Arkh 74, 23-26.

Kofler, S., Nickel, T. \& Weis, M. (2005). Role of cytokines in cardiovascular diseases: a focus on endothelial responses to inflammation. Clin Sci 108, 205-213.

Larsen, C. G., Anderson, A. O., Appella, E., Oppenheim, J. J. \& Matsushima, K. (1989). The neutrophil-activating protein (NAP-1) is also chemotactic for T lymphocytes. Science 243, 1464-1466.

Lepidi, H., Fournier, P. E. \& Raoult, D. (2006). Histologic features and immunodetection of African tick-bite fever eschar. Emerg Infect Dis 12, 1332-1337.

Liu, S. F. \& Malik, A. B. (2006). NF- $\kappa$ B activation as a pathological mechanism of septic shock and inflammation. Am J Physiol Lung Cell Mol Physiol 290, L622-L645.

McLeod, M. P., Qin, X., Karpathy, S. E., Gioia, J., Highlander, S. K., Fox, G. E., McNeill, T. Z., Jiang, H., Muzny, D. \& other authors (2004). Complete genome sequence of Rickettsia typhi and comparison with sequences of other rickettsiae. J Bacteriol 186, 5842-5855.

Ogata, H., Audic, S., Renesto-Audiffren, P., Fournier, P. E., Barbe, V., Samson, D., Roux, V., Cossart, P., Weissenbach, J. \& other authors (2001). Mechanisms of evolution in Rickettsia conorii and $R$. prowazekii. Science 293, 2093-2098. 
Olano, J. P. (2005). Rickettsial infections. Ann N Y Acad Sci 1063, 187-196.

Policastro, P. F., Peacock, M. G. \& Hackstadt, T. (1996). Improved plaque assays for Rickettsia prowazekii in Vero 76 cells. J Clin Microbiol 34, 1944-1948.

Radulovic, S., Price, P., Beier, M., Gaywee, J., Macaluso, J. \& Azad, A. (2002). Rickettsia-macrophage interactions: host cell responses to Rickettsia akari and Rickettsia typhi. Infect Immun 70, 2576-2582.

Regnery, R. L., Spruill, C. L. \& Plikaytis, B. D. (1991). Genotypic identification of rickettsiae and estimation of intraspecies sequence divergence for portions of two rickettsial genes. J Bacteriol 173, 1576-1589.

Rondaij, M. G., Bierings, R., Kragt, A., van Mourik, J. A. \& Voorberg, J. (2006). Dynamics and plasticity of Weibel-Palade bodies in endothelial cells. Arterioscler Thromb Vasc Biol 26, 1002-1007.

Rydkina, E., Sahni, S. K., Santucci, L. A., Turpin, L. C., Baggs, R. B. \& Silverman, D. J. (2004). Selective modulation of antioxidant enzyme activities in host tissues during Rickettsia conorii infection. Microb Pathog 36, 293-301.

Rydkina, E., Silverman, D. J. \& Sahni, S. K. (2005). Selective activation of p38 stress-activated protein kinase during Rickettsia rickettsii infection of human endothelial cells: role in the induction of chemokine response. Cell Microbiol 7, 1519-1530.

Sahni, S. K. (2007). Endothelial cell infection and hemostasis. Thromb Res 119, 531-549.

Sahni, S. K., Van Antwerp, D. J., Eremeeva, M. E., Silverman, D. J., Marder, V. J. \& Sporn, L. A. (1998). Proteasome-independent activation of nuclear factor $\kappa \mathrm{B}$ in cytoplasmic extracts from human endothelial cells by Rickettsia rickettsii. Infect Immun 66, 1827-1833.

Sahni, S. K., Rydkina, E., Joshi, S. G., Sporn, L. A. \& Silverman, D. J. (2003). Interactions of Rickettsia rickettsii with endothelial nuclear factor- $\kappa \mathrm{B}$ in a 'cell-free' system. Ann N Y Acad Sci 990, 635-641.

Sessler, C. N. \& Fowler, A. A., III (1996). Fulminant Rocky Mountain spotted fever: a hemophagocytic syndrome? Crit Care Med 24, 365.

Sessler, C. N., Schwartz, M., Windsor, A. C. \& Fowler, A. A., III (1995). Increased serum cytokines and intercellular adhesion molecule-1 in fulminant Rocky Mountain spotted fever. Crit Care Med 23, 973-976.

Shi, R. J., Simpson-Haidaris, P. J., Lerner, N. B., Marder, V. J., Silverman, D. J. \& Sporn, L. A. (1998). Transcriptional regulation of endothelial cell tissue factor expression during Rickettsia rickettsii infection: involvement of the transcription factor NF- $\kappa \mathrm{B}$. Infect Immun 66, 1070-1075

Sporn, L. A. \& Marder, V. J. (1996). Interleukin-1 $\alpha$ production during Rickettsia rickettsii infection of cultured endothelial cells: potential role in autocrine cell stimulation. Infect Immun 64, 1609-1613.
Sporn, L. A., Shi, R. J., Lawrence, S. O., Silverman, D. J. \& Marder, V. J. (1991). Rickettsia rickettsii infection of cultured endothelial cells induces release of large von Willebrand factor multimers from Weibel-Palade bodies. Blood 78, 2595-2602.

Sporn, L. A., Lawrence, S. O., Silverman, D. J. \& Marder, V. J. (1993). E-selectin-dependent neutrophil adhesion to Rickettsia rickettsiiinfected endothelial cells. Blood 81, 2406-2412.

Sporn, L. A., Sahni, S. K., Lerner, N. B., Marder, V. J., Silverman, D. J., Turpin, L. C. \& Schwab, A. L. (1997). Rickettsia rickettsii infection of cultured human endothelial cells induces NF- $\kappa$ B activation. Infect Immun 65, 2786-2791.

Taub, D. D., Anver, M., Oppenheim, J. J., Longo, D. L. \& Murphy, W. J. (1996). T lymphocyte recruitment by interleukin-8 (IL-8). IL-8induced degranulation of neutrophils releases potent chemoattractants for human T lymphocytes both in vitro and in vivo. J Clin Invest 97, 1931-1941.

Teysseire, N., Arnoux, D., George, F., Sampol, J. \& Raoult, D. (1992). von Willebrand factor release and thrombomodulin and tissue factor expression in Rickettsia conorii-infected endothelial cells. Infect Immun 60, 4388-4393.

Teysseire, N., Boudier, J. A. \& Raoult, D. (1995). Rickettsia conorii entry into Vero cells. Infect Immun 63, 366-374.

Valbuena, G. \& Walker, D. H. (2006). The endothelium as a target for infections. Annu Rev Pathol Mech Dis 1, 171-198.

Valbuena, G., Bradford, W. \& Walker, D. H. (2003). Expression analysis of the T-cell-targeting chemokines CXCL9 and CXCL10 in mice and humans with endothelial infections caused by rickettsiae of the spotted fever group. Am J Pathol 163, 1357-1369.

Valbuena, G., Jordan, J. M. \& Walker, D. H. (2004). T cells mediate cross-protective immunity between spotted fever group rickettsiae and typhus group rickettsiae. J Infect Dis 190, 1221-1227.

Viatour, P., Merville, M. P., Bours, V. \& Chariot, A. (2005). Phosphorylation of NF- $\kappa \mathrm{B}$ and $\mathrm{I} \kappa \mathrm{B}$ proteins: implications in cancer and inflammation. Trends Biochem Sci 30, 43-52.

Vitale, G., Mansueto, S., Gambino, G., Mocciaro, C., Spinelli, A., Rini, G. B., Affronti, M., Chifari, N., La Russa, C. \& other authors (2001). The acute phase response in Sicilian patients with boutonneuse fever admitted to hospitals in Palermo, 1992-1997. J Infect 42, 33-39.

Walker, D. H. \& Yu, X. J. (2005). Progress in rickettsial genome analysis from pioneering of Rickettsia prowazekii to the recent Rickettsia typhi. Ann N Y Acad Sci 1063, 13-25.

Xiao, C. \& Ghosh, S. (2005). NF- $\kappa$ B, an evolutionarily conserved mediator of immune and inflammatory responses. Adv Exp Med Biol 560, 41-45. 